\title{
Study on the Thick-Walled Pipe Ultrasonic Signal Enhancement of Modified S-Transform and Singular Value Decomposition
}

\author{
Haichao Cai, ${ }^{1,2}$ Chunguang Xu, ${ }^{1}$ Shiyuan Zhou, ${ }^{1}$ Hongjuan Yan, ${ }^{1}$ and Liu Yang ${ }^{1}$ \\ ${ }^{1}$ Key Laboratory of Fundamental Science for Advanced Machining, Beijing Institute of Technology, Beijing 100081, China \\ ${ }^{2}$ School of Mechatronics Engineering, Henan University of Science and Technology, Luoyang 471000, China \\ Correspondence should be addressed to Haichao Cai; chclovely99@eyou.com
}

Received 29 August 2014; Revised 16 December 2014; Accepted 25 December 2014

Academic Editor: Giuseppe Rega

Copyright (c) 2015 Haichao Cai et al. This is an open access article distributed under the Creative Commons Attribution License, which permits unrestricted use, distribution, and reproduction in any medium, provided the original work is properly cited.

\begin{abstract}
When detecting the ultrasonic flaw of thick-walled pipe, the flaw echo signals are often interrupted by scanning system frequency and background noise. In particular when the thick-walled pipe defect is small, echo signal amplitude is often drowned in noise signal and affects the extraction of defect signal and the position determination accuracy. This paper presents the modified Stransform domain singular value decomposition method for the analysis of ultrasonic flaw echo signals. By changing the scale rule of Gaussian window functions with S-transform to improve the time-frequency resolution. And the paper tries to decompose the singular value decomposition of time-frequency matrix after the S-transform to determine the singular entropy of effective echo signal and realize the adaptive filter. Experiments show that, using this method can not only remove high frequency noise but also remove the low frequency noise and improve the signal-to-noise ratio of echo signal.
\end{abstract}

\section{Introduction}

In petroleum, electric power, nuclear industry department, there are a large number of pipelines. In order to ensure the efficiency and benefit of pipeline operation, which can withstand high pressure and temperature, these pipelines tend to have larger pipe wall thickness. Practice shows that the pipes often have flaws such as crack, corrosion in the long run, along with the change of material properties. Seriously, it can endanger the safety of the pipeline $[1,2]$. Therefore, testing the pipeline condition timely is important. Commonly used pipeline detection methods, such as magnetic powder, penetrant, radiography, and ultrasonic, are commonly used for pipe detection, but ultrasonic test method is widely used for its simple principle and harmlessness to human body [3]. However, when detecting the ultrasonic flaw for the thickwalled pipes, due to the scattering and attenuation, echo signal is a nonstationary random signal, which contains information related to the defect. By extracting the information of pipeline defect, it can judge whether the pipelines have flaws or not. But when scanning the power frequency interference of the system, various doping factors such as environmental noise mixed in the echo signal, it causes great influence to the defect judgment of the gate. Therefore, noise suppression and the effective extraction of echo to realize the accurate recognition are a problem worthy of further research.

Ultrasonic signal processing technology has made great progress. The correlated analysis, Fourier transform, shorttime Fourier transform (STFT), wavelet transform (WT), and pseudo Wigner transform method have been used for ultrasonic signal analysis and processing. These methods of the ultrasonic signal processing show the corresponding advantages [4-8]. But the correlated analysis is just the time domain analysis; and Fourier transform is just frequency domain analysis; the signal short-time steady states, which STFT need, cannot make it effectively distinguish the multiple echo signal submerged in noise. Moreover, STFT uses the constant sliding window, and it cannot accurately decompose low frequency signal whose cycle is bigger than the time window; although WT can effectively reflect the signal of low frequency and high frequency components, it looks for suitable wavelet basis function which is not close connected with the Fourier spectrum item; the pseudo Wigner distribution is 
not linear transformation; the response signal spectrum will appear harmful cross terms [9-12].

S-transform is proposed by Stockwell et al. [13] which is an important tool to study the distribution of nonstationary signal's time frequency. The following scholars promote it to the generalized S-transform. It is the product of STFT and WT joint development, which not only overcomes the fixed STFT window frequency and the absence of WT signal phase information, but also absorbs the characteristic of wavelet multiresolution, for example, the characteristics of nondestructivity, no cross interference of Cohen. It does not need signal and measuring system and does not have to satisfy admissibility conditions and so forth $[14,15]$. This paper applies the modified S-transform and singular value decomposition with the signal analysis of thick-walled pipe, through controlling the noise to get better extraction of ultrasonic echo signal time-frequency information of purposes, to achieve effective testing.

This paper is organized as follows. Section 2 describes the basic principle of S-transform. In Section 3, we elaborate a mathematical model of ultrasonic signals and then present an enhancement algorithm based on modified S-transform and singular values decomposition. Section 4 utilizes ultrasonic simulation signal with different noise level and experiment signal to validate the performance of our presented algorithm and gives results and analysis. Conclusions are presented in Section 5 .

\section{S-Transform Theories}

2.1. S-Transform. The known frequency domain of $x(t)$ is expressed as the Fourier transform:

$$
X(f)=\int_{-\infty}^{\infty} x(t) e^{-j 2 \pi f t} d t
$$

where $f$ is represented as frequency, because Fourier transforms can only reflect the signal frequency domain information. The frequency can both reflect the signal frequency domain information and reflect the signal time-frequency representation as follows:

$$
\operatorname{FT}(\tau, f)=\int_{-\infty}^{\infty} x(t) w(\tau-t) e^{-j 2 \pi f t} d t
$$

$w(t)$ is the Gaussian window function:

$$
w(t, \varphi)=\frac{1}{\sqrt{2 \pi} \varphi} e^{-t^{2} / 2 \varphi^{2}}
$$

Equation (2) is the STFT; $\varphi$ is frequency scale regulation factor; $\tau$ is time shift factor; if substituting $\varphi=1 / f$ in (3), then it can get

$$
w(t, f)=\frac{|f|}{\sqrt{2 \pi}} e^{-f^{2} t^{2} / 2}
$$

Equation (4) is substituted in (5); it can get

$$
S(\tau, f)=\int_{-\infty}^{\infty} x(t) \frac{|f|}{\sqrt{2 \pi}} e^{-f^{2}(t-\tau)^{2} / 2} e^{-j 2 \pi f t} d t
$$

Equation (5) is the S-transform definition given by Pinnegar and Mansinha [16], the window function $w(t)$ of S-transform should satisfy

$$
\int_{-\infty}^{\infty} w(t, f) d t=1
$$

Therefore, by the time integral on the S-transform, we can get

$$
\begin{aligned}
\int_{-\infty}^{\infty} S(\tau, f) d t & =\int_{-\infty}^{\infty} \int_{-\infty}^{\infty} x(t) \frac{|f|}{\sqrt{2 \pi}} e^{-f^{2}(t-\tau)^{2} / 2} e^{-j 2 \pi f t} d t \\
& =X(f) .
\end{aligned}
$$

From (7) it can be got that the inverse transformation of Stransform [17] can be obtained from $X(f)$ inverse Fourier transform

$$
\int_{-\infty}^{\infty} X(f) e^{j 2 \pi f t} d f=\int_{-\infty}^{\infty}\left(\int_{-\infty}^{\infty} S(\tau, f) d t\right) e^{j 2 \pi f t} d f=x(t)
$$

2.2. Modified S-Transform. If introducing two regulatory factors $a, b$ in S-transform Gauss function to control time and frequency resolution, then window function $w(t)$ can be changed into

$$
w(t, f, a, b)=\frac{a|f|^{b}}{\sqrt{2 \pi}} e^{-a^{2} f^{2 b} t^{2} / 2}
$$

Equation (9) is substituted in (5); it can get modified Stransform [18]:

$$
S(\tau, f, a, b)=\int_{-\infty}^{\infty} x(t) \frac{a|f|^{b}}{\sqrt{2 \pi}} e^{-a^{2} f^{2 b}(t-\tau)^{2} / 2} e^{-j 2 \pi f t} d t .
$$

Based on Fourier transform properties, (10) time domain can be changed into frequency domain:

$$
S(\tau, f, a, b)=\int_{-\infty}^{\infty} X(\eta+f) e^{-2 \pi^{2} \eta^{2} / f^{2}} e^{i 2 \pi \eta \tau} d \eta
$$

\section{Modified S-Transform and Singular Value Decomposition}

Set the original signal sampled discrete time series $f\left(t_{j}\right)$, noise discrete time series $\sigma\left(t_{j}\right)$, mathematical model for detecting the actual signal as follows:

$$
x\left(t_{j}\right)=f\left(t_{j}\right)+\sigma\left(t_{j}\right) \quad(j=0,1,2, \ldots, N-1) .
$$


Improving the discrete time sequence $x\left(t_{j}\right)$, S-transform can get

$$
\begin{aligned}
S[p, q]= & \sum_{j=0}^{N-1} x\left(t_{j}\right) \frac{a|q|^{b}}{\sqrt{2 \pi} N} e^{-a^{2} q^{2 b}(p-j)^{2} / 2 N^{2}} e^{-i 2 \pi j a q^{b} / N} \\
= & \sum_{j=0}^{N-1} f\left(t_{j}\right) \frac{a|q|^{b}}{\sqrt{2 \pi} N} e^{-a^{2} q^{2 b}(p-j)^{2} / 2 N^{2}} e^{-i 2 \pi j a q^{b} / N} \\
& +\sum_{j=0}^{N-1} \sigma\left(t_{j}\right) \frac{a|q|^{b}}{\sqrt{2 \pi} N} e^{-a^{2} q^{2 b}(p-j)^{2} / 2 N^{2}} e^{-i 2 \pi j a q^{b} / N}, \\
A_{(p q) j}= & \sum_{j=0}^{N-1} \frac{a|q|^{b}}{\sqrt{2 \pi} N} e^{-a^{2}(p-j)^{2} q^{2 b} / 2 N^{2}} e^{-i j 2 \pi a q^{b} / N} .
\end{aligned}
$$

$p, q=0,1,2, \ldots, N-1 ; A_{(p q) j}$ is the transform coefficients after modified S-transform; for coefficient $A_{(p q) j}$, it can use matrix

$$
A_{(p q) j}=\left[\begin{array}{cccc}
A_{11} & A_{12} & \cdots & A_{1 N} \\
A_{21} & A_{22} & \cdots & A_{2 N} \\
\vdots & \vdots & \cdots & \vdots \\
A_{N 1} & A_{N 2} & \cdots & A_{N N} \\
A_{(N+1) 1} & A_{(N+1) 2} & \cdots & A_{(N+1) N} \\
\vdots & \vdots & \cdots & \vdots \\
A_{(p q) 1} & A_{(p q) 2} & \cdots & A_{(p q) N}
\end{array}\right] .
$$

The coefficient matrix contains not only the original time series information but also the noise time sequence information. Because the noise signal energy distribution is scattered, the adjustable time-frequency factors $a, b$ make the signal energy concentrated and then decompose the modified S-transform matrix by using the singular value. The decomposed singular embodies the useful signal and noise. The large singular values mainly reflect the useful signal; the rest of singular values reflect noise and analyze the singular entropy to determine the optimal order number and reconstruct the original signal. The algorithm steps of modified S-transform and singular value decompositions are as follows.

(1) Change the thick-walled pipes ultrasonic signal by using the modified S-transform, in order to determine the value of time-frequency regulator.

(2) Decompose the $N \times N$ time-frequency matrix by using singular value decomposition; the definition of singular value decomposition can be got from [19]

$$
S_{N \times N}=W_{N \times l} \Lambda_{l \times l} V_{l \times N}^{T} .
$$

$W$ and $V^{T}$ are feature vector and indicate signal time frequency and frequency information

$$
\Lambda=\left|\begin{array}{cccc}
\delta_{1} & 0 & \cdots & 0 \\
0 & \delta_{2} & 0 & \vdots \\
\vdots & 0 & \ddots & 0 \\
0 & \cdots & 0 & \delta_{l}
\end{array}\right| .
$$

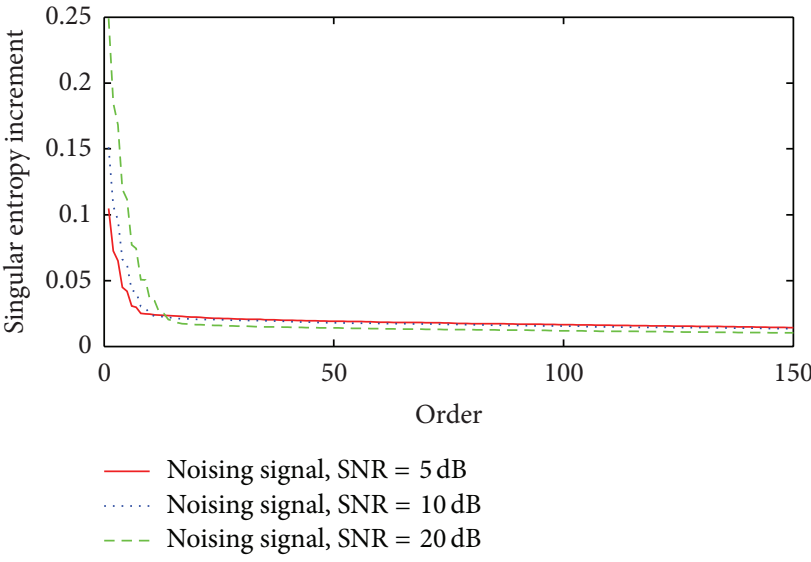

FIGURE 1: The variation tendency of singular entropy increment with the variation of the order.

The diagonal element of (16) satisfies $\delta_{1} \geq \delta_{2} \geq \cdots \geq \delta_{l} \geq 0$; all the diagonal elements of the matrix $\Lambda$ are called singular value of matrix $S$. After the singular value decomposition, the singular value number of zeros is bigger and bigger, which shows the signal-to-noise ratio is good.

(3) Compute (17) by using the signal singular entropy increment

$$
\Delta E_{r}=\sum_{i=1}^{r}-\left(\frac{\delta_{i}}{m}\right) \log \left(\frac{\delta_{i}}{m}\right)
$$

$m=\sum_{j=1}^{l} \delta_{j}, r \leq l, r$ is the order of singular entropy, and $l$ is the total number of singular value.

(4) Compute the ratio between $r+1$ and singular entropy $\Delta E_{r+1} / \Delta E_{r}$. Taking increment ratio of singular entropy as the threshold for the trade-offs of singular value, in terms of filter accuracy, while signal of interest is easily filtered if the chosen ratio is too small, it is hard to be effectively filtered if the ratio is too large. Figure 1 shows the variation tendency of singular entropy increment for ultrasonic signal of different SNR with the variation of the order. It can be seen that at low order, with the increase of signal-to-noise ratio (SNR), the singular entropy increment also increases. At high order, however, the singular entropy increment decreases with the increase of SNR. Regardless of SNR, with the order increasing, singular entropy increment is decreasing and approaching saturation. This means that increment ratio of singular entropy is also approaching saturation; when the increment ratio of singular entropy is equal to 0.9 , the signal mainly contains useful signal and most of the noise signals are removed. Therefore, the singular entropy increment ratio 0.9 can be chosen as a threshold. Figure 2 is the arithmetic flowchart. If the ratio of singular entropy is greater than 0.9 , then make singular value which is greater than or equal to $r+1$ order equal zero and make up the novelty spectrum matrix $\Lambda^{\prime}$, according to formula (15) to obtain new data matrix. The new data matrix inverses S-transform to obtain the denoising ultrasonic signal of thick-walled pipe. 


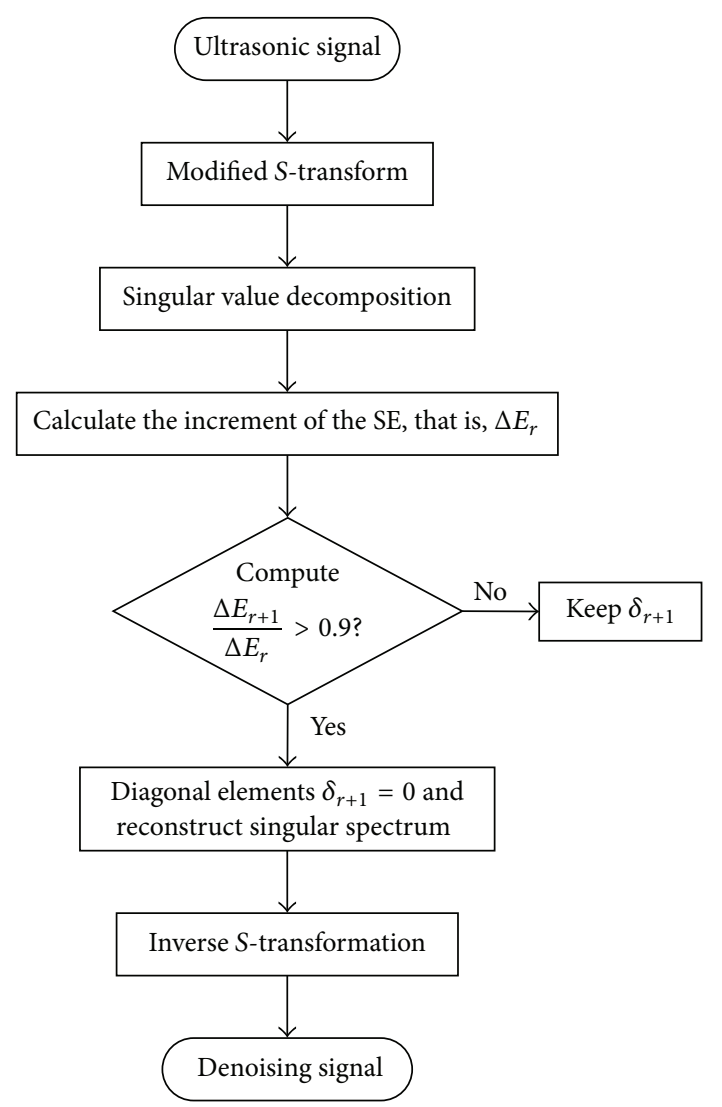

FIGURE 2: The flowcharts of modified S-transform singular values decomposition.

\section{Simulation and Experimental Analysis}

To verify the denoising effect and robustness of the modified S-transform and singular value decomposition algorithm, the paper selects ultrasonic signal submerged at different noising signal level and selects the experimental signal to analyze and to show that it can be applied to ultrasonic nondestructive testing.

4.1. Simulation Study. Formula (18) generates the pure ultrasound signal without noise and adds Gauss white noise signal with different signal ratio:

$$
\begin{array}{r}
x(t)=A_{i} e^{-\alpha\left(t-\tau_{i}\right)^{2}} \times \cos \left(2 \pi f_{i}\left(t-\tau_{i}\right)\right)+n(t) \\
(i=1,2,3, \ldots),
\end{array}
$$

where $A_{i}, \tau_{i}, f_{i}$ are the $i$ th signal magnitude, the arrival of time signals, and signal center frequency; $\alpha$ is signal bandwidth factor; $n(t)$ is Gauss white noise. Figure 3 is noisefree ultrasonic simulation signals, which simulate ultrasonic echo signals parameters $A_{1}=10, \alpha=10(\mathrm{MHz})^{2}, f_{1}=$ $f_{2}=5 \mathrm{MHz}, \tau_{1}=0.6 \mu \mathrm{s}, A_{2}=6, \tau_{2}=1.8 \mu \mathrm{s}$. Denoising

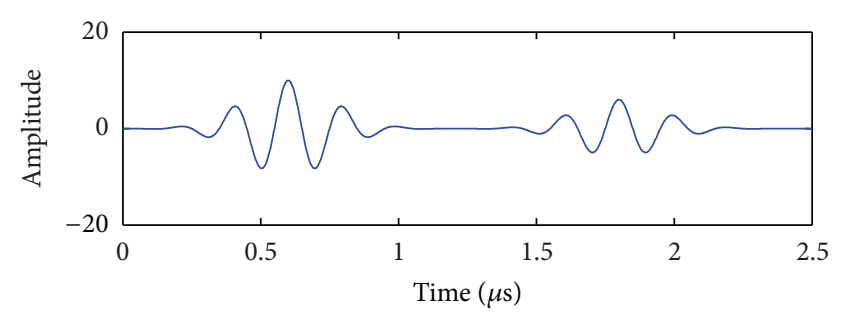

FIGURE 3: Noise-free ultrasonic simulation signals.

TABLE 1: The denoising result of ultrasonic signal at different noise levels.

\begin{tabular}{lccc}
\hline $\begin{array}{l}\text { Evaluation } \\
\text { indicators }\end{array}$ & The indicator value after denoising at different noise \\
& $20 \mathrm{db}$ & $10 \mathrm{db}$ & $5 \mathrm{db}$ \\
\hline $\mathrm{SNR} / \mathrm{db}$ & 21.1278 & 13.4793 & 11.8967 \\
$\mathrm{SNRG} / \mathrm{db}$ & 1.1278 & 3.4793 & 6.8967 \\
\hline
\end{tabular}

performance of algorithm was measured with the signal-tonoise ratio (SNR) and signal-to-noise ratio gain (SNRG). The results are shown in Table 1. The formula of SNR is as follows:

$$
\mathrm{SNR}=10 \lg \frac{\sum_{i=1}^{N} f^{2}(i)}{\sum_{i=1}^{N}(\sigma(i)-f(i))^{2}} .
$$

$\sigma(i)$ is discrete noise signals, $f(i)$ is discrete pure signals, $N$ is the length of signals. SNRG is difference between the SNR before denoising and that after denoising.

The results of denoising simulated ultrasonic signals with random Gauss white noise in different SNR by modified $\mathrm{S}$-transform and singular value decomposition is shown in Figures 4,5 , and 6 . Time Regulation factor in the modified Stransform is $a=2, b=0.9$. Through the analysis of singular entropy, the number of singular value is identified as 2 .

The results are shown in Table 1 . The algorithm proves better denoising effects when the SNR are $20 \mathrm{db}, 10 \mathrm{db}$, and $5 \mathrm{db}$. With the noise level increasing, the SNR gain is also increasing and the improvement of SNR due to the algorithm is more significant. This indicates that the algorithm is of strong denoising ability to improve the SNR value at high noise level. But when the SNR is low, although most of the noise can be eliminated, high frequency noise attached to the ultrasonic signal still has few residues.

4.2. Experimental Results. The paper selects the oil drill collar to conduct the actual ultrasonic defect detection experiments. The drill collar is made by chromium molybdenum alloy structure steel, the outer diameter is $79.4 \mathrm{~mm}$, the wall thickness is $47.6 \mathrm{~mm}$, the length is $60 \mathrm{~mm}$, and the inner surface of drill collar has a $60 \mathrm{~mm}$ long, $1 \mathrm{~mm}$ wide, and $1.5 \mathrm{~mm}$ deep rectangular groove defect. The experimental detection system is made of water immersion focusing ultrasonic probe whose center frequency is $5 \mathrm{MHz}$, with AD-IPR1210 pulsed ultrasonic transceiver, 4104B four-channel Tektronix digital oscilloscope, computer, and so forth. Figure 7 is the drill collar ultrasonic detecting system schematic diagram. 

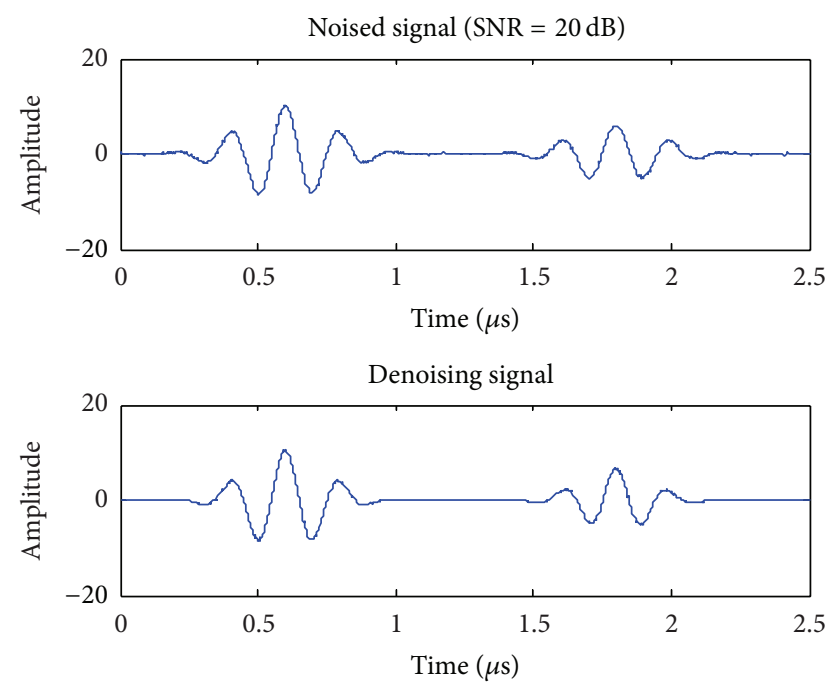

FIgURE 4: SNR = $20 \mathrm{db}$ noised and denoising signal waveforms.
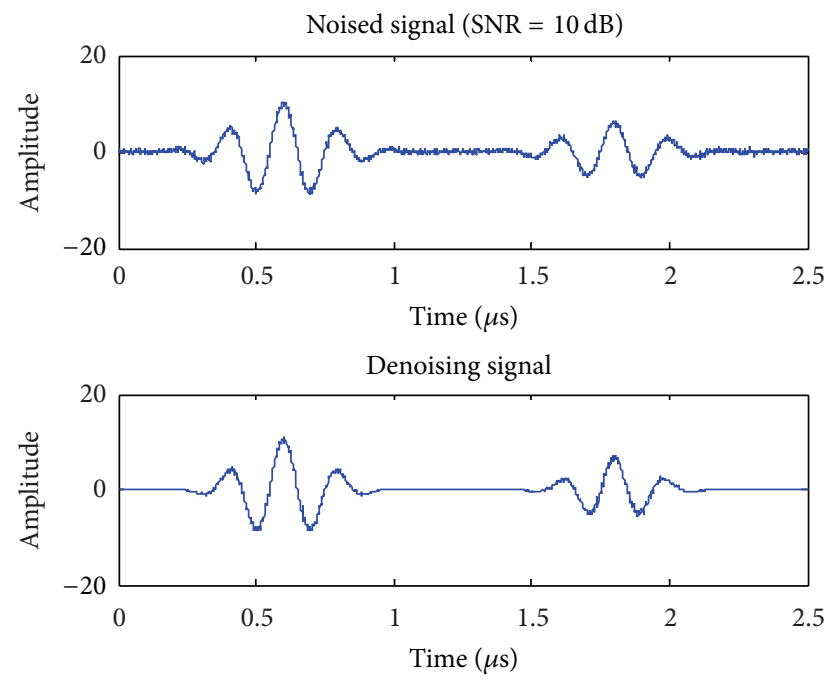

FIGURE 5: SNR $=10 \mathrm{db}$ noised and denoising signal waveforms.
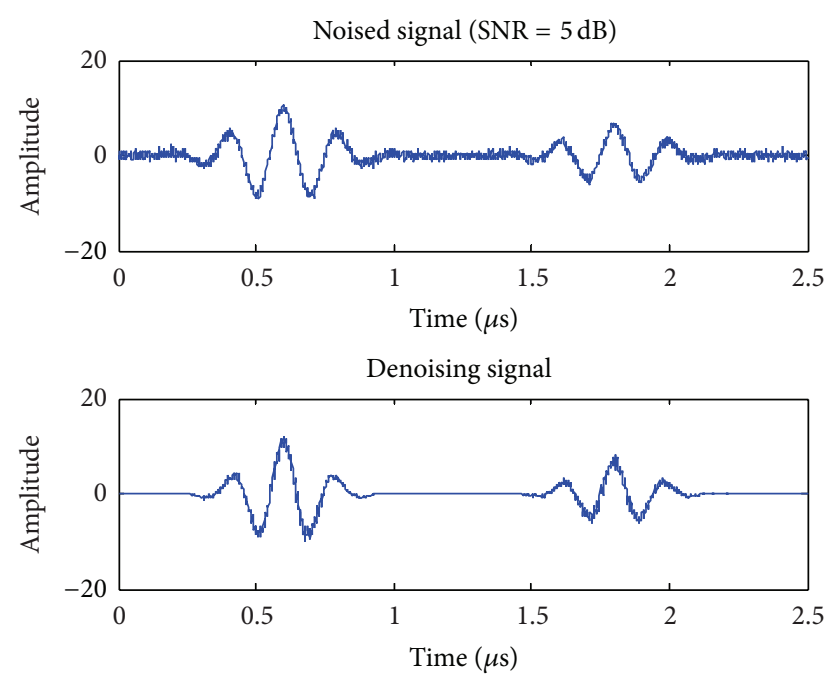

FIGURE 6: $\mathrm{SNR}=5 \mathrm{db}$ noised and denoising signal waveforms.

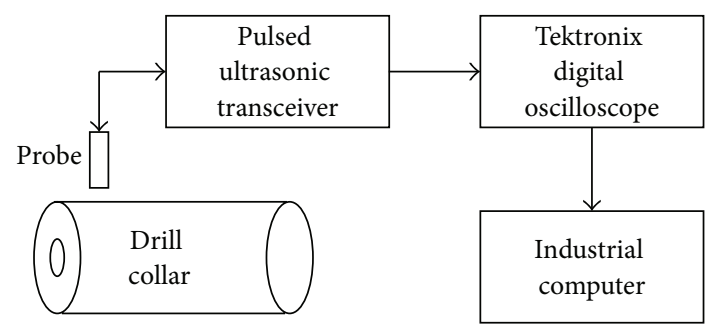

(a) Detecting system diagram

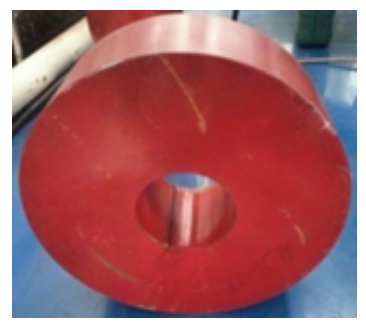

(b) Drill collar

FIGURE 7: Drill collar ultrasonic detecting system.

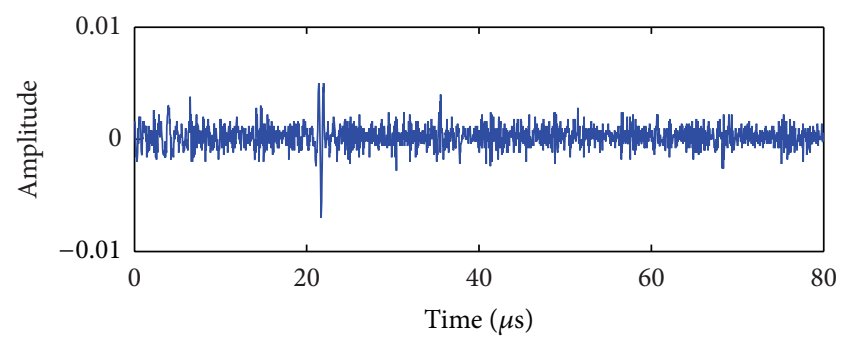

FIgURE 8: Drill collars flaw echo signal time waveforms.

The experiment chooses the sampling frequency $25 \mathrm{MHz}$. Figure 8 is the flaw echo signal time domain waveforms of drill collars. It can be seen from the diagram that waveforms are almost overwhelmed by a lot of noise, especially when the flaw sizes are small. It will have a great difficulty in scanning threshold and influences defect detection sensitivity and resolution.

The ultrasonic signals in wavelet threshold denoising of Figure 8 are shown in Figure 10, compared with Figure 8, and by using wavelet packet default threshold method have reduced the noise signal, but echo is not evident in the denoising signal details; waveform shows many peaks which cannot effectively distinguish echo and cannot gate position precisely.

Choose the modified S-transform time-frequency factors $a, b$, respectively, as 2 and 1 to make S-transform for the ultrasonic echo signal; then compute the singular value decomposition of transform time-frequency matrix. Based on the singular entropy increment ratio, to determine the optimal singular entropy as order 2, which retains the first two of all singular values, the rest is value zero. Figure 9 is ultrasonic signal modified S-transform and SVD denoising waveforms. Compared with Figures 9 and 10, using the modified S-transform and singular value decomposition algorithm 


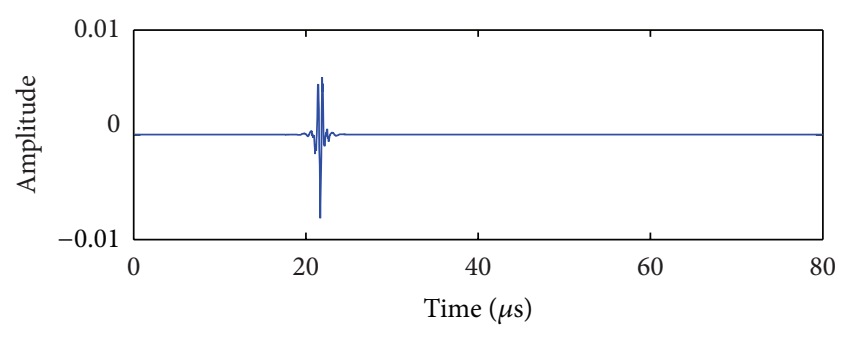

FIGURE 9: Ultrasonic signal modified S-transform and SVD denoising waveforms.

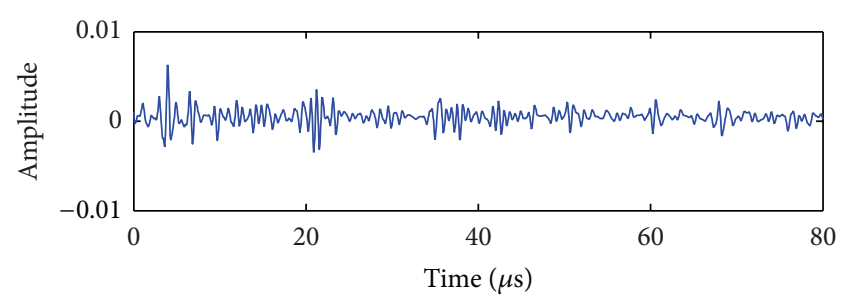

FIgURE 10: Ultrasonic signal wavelet packet default threshold denoising waveforms.

to reduce the noise interference, the defect echo signal peak is clearly visible, which is in favor of gate settings and qualitative and quantitative analysis.

\section{Conclusions}

According to the nonstationary of thick-walled pipe ultrasonic echo signals, combined with good sensitivity to the noise in the original signal of ultrasonic echo signal, this paper proposes the modified S-transform and singular value decomposition denoising algorithm model. Based on the basic theory of S-transform, this paper makes the singular value decomposition formula and calculates the signal singular entropy, the increment ratio of singular entropy as the threshold of discrimination, simulation, and experiment to verify the algorithm.

The results show that compared with the commonly used wavelet threshold denoising method, the modified Stransform and singular value decomposition method can effectively reduce high frequency and low frequency noise and improve signal-to-noise ratio, more suitable for thickwalled pipe ultrasonic scanning signal analysis and denoising, in order to prevent the leakage of pipeline defects, the miscarriage of justice, which established a good foundation.

\section{Conflict of Interests}

The authors declare that there is no conflict of interests regarding the publication of this paper.

\section{Acknowledgment}

This research work is supported by the National Natural Science Fund of China (no. 50975028).

\section{References}

[1] J. Szelążek, P. Gutkiewicz, P. Grzywna, and S. Mackiewicz, "Ultrasonic measurement of thick-walled pipe diameter changes," Journal of Pressure Vessel Technology, Transactions of the ASME, vol. 136, no. 4, Article ID 041408, 2014.

[2] N. P. Aleshin, Y. L. Gobov, A. V. Mikhailov, Y. G. Smorodinskii, and M. M. Syrkin, "Automatic ultrasonic inspection of largediameter pipes," Russian Journal of Nondestructive Testing, vol. 50, no. 3, pp. 133-140, 2014.

[3] M. Moles, N. Dubé, S. Labbé, and E. Ginzel, "Review of ultrasonic phased arrays for pressure vessel and pipeline weld inspections," Journal of Pressure Vessel Technology-Transactions of the ASME, vol. 127, no. 3, pp. 351-356, 2005.

[4] V. K. Kachanov, V. G. Kartashev, and V. P. Popko, "Application of signal processing methods to ultrasonic non-destructive testing of articles with high structural noise," Nondestructive Testing and Evaluation, vol. 17, no. 1, pp. 15-40, 2001.

[5] W. T. Kuang and A. S. Morris, "Using short-time Fourier transform and wavelet packet filter banks for improved frequency measurement in a Doppler robot tracking system," IEEE Transactions on Instrumentation and Measurement, vol. 51, no. 3, pp. 440-444, 2002.

[6] J. L. San Emeterio, E. Pardo, A. Ramos, and M. A. Rodriguez, "Ultrasonic grain noise reduction using wavelet processing, an analysis of threshold selection rules," Berlin, 2006.

[7] S.-P. Song and P.-W. Que, "Wavelet based noise suppression technique and its application to ultrasonic flaw detection," Ultrasonics, vol. 44, no. 2, pp. 188-193, 2006.

[8] M. A. Rodríguez, J. L. San Emeterio, J. C. Lázaro, and A. Ramos, "Ultrasonic flaw detection in NDE of highly scattering materials using wavelet and Wigner-Ville transform processing," Ultrasonics, vol. 42, no. 1-9, pp. 847-851, 2004.

[9] R. Raišutis, O. Tumšys, R. Kažys, and L. Mažeika, "A comparative study of time-frequency analysis techniques in the case of signal processing for ultrasonic NDT,' Insight, vol. 50, no. 11, pp. 628-633, 2008.

[10] R. Drai, M. Khelil, and A. Benchaala, "Time frequency and wavelet transform applied to selected problems in ultrasonics NDE," NDT \& E International, vol. 35, no. 8, pp. 567-572, 2002.

[11] A. Benammar, R. Drai, and A. Guessoum, "Ultrasonic flaw detection using threshold modified S-transform," Ultrasonics, vol. 54, no. 2, pp. 676-683, 2014.

[12] B. Birendra, K. D. Pradipta, and B. Milan, "Time frequency analysis and FPGA implementation of modified S-tansform for De-noising," International Journal of Signal Processing, Image Processing and Pattern Recognition, vol. 4, no. 2, pp. 119-135, 2011.

[13] R. G. Stockwell, L. Mansinha, and R. P. Lowe, "Localization of the complex spectrum: the S transform," IEEE Transactions on Signal Processing, vol. 44, no. 4, pp. 998-1001, 1996.

[14] L. Mansinha, R. G. Stockwell, and R. P. Lowe, "Pattern analysis with two-dimensional spectral localisation: applications of twodimensional S transforms," Physica A: Statistical Mechanics and its Applications, vol. 239, no. 1-3, pp. 286-295, 1997.

[15] P. D. McFadden, J. G. Cook, and L. M. Forster, "Decomposition of gear vibration signals by the generalised $S$ transform," Mechanical Systems and Signal Processing, vol. 13, no. 5, pp. 691708, 1999.

[16] C. R. Pinnegar and L. Mansinha, "The S-transform with windows of arbitrary and varying shape," Geophysics, vol. 68, no. 1, pp. 381-385, 2003. 
[17] M. Schimmel and J. Gallart, "The inverse $S$-transform in filters with time-frequency localization," IEEE Transactions on Signal Processing, vol. 53, no. 11, pp. 4417-4422, 2005.

[18] R. G. Stockwell, "Why use the S-transform?" in PseudoDifferential Operators: Partial Differential Equations and TimeFrequency Analysis, vol. 52 of Fields Institute communications, pp. 279-309, American Mathematical Society, 2007.

[19] W.-X. Yang and P. W. Tse, "Development of an advanced noise reduction method for vibration analysis based on singular value decomposition," NDT and E International, vol. 36, no. 6, pp. 419-432, 2003. 


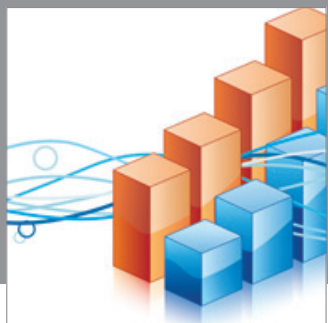

Advances in

Operations Research

mansans

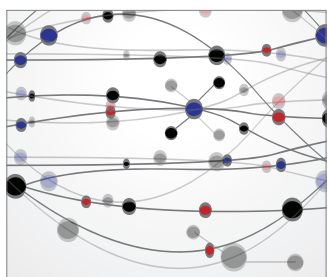

The Scientific World Journal
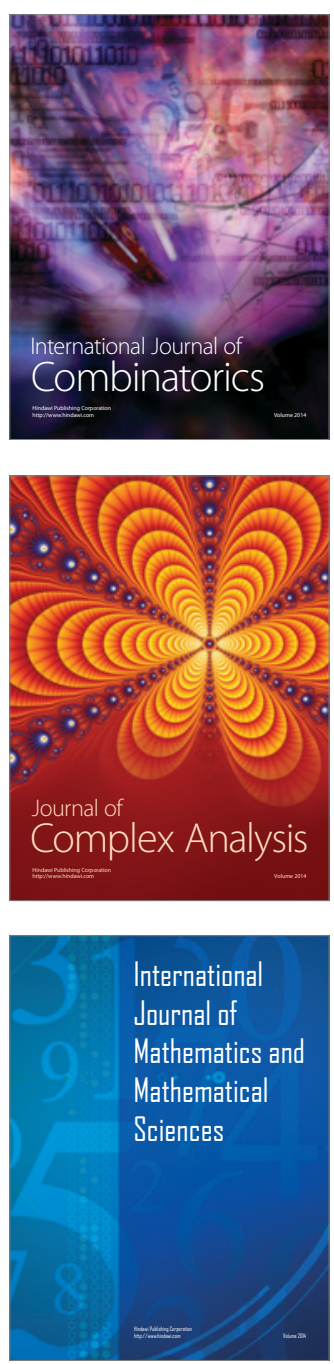
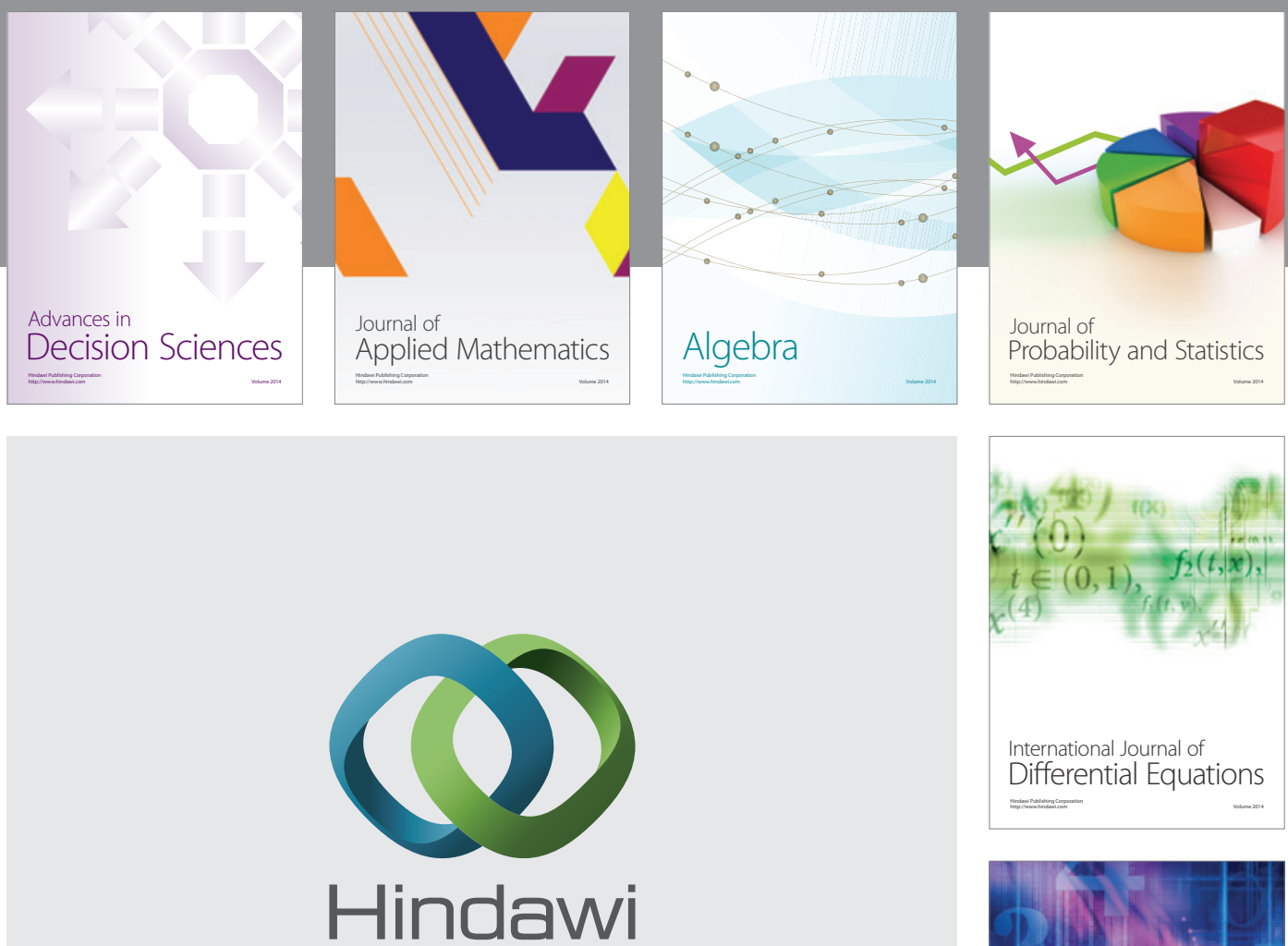

Submit your manuscripts at http://www.hindawi.com
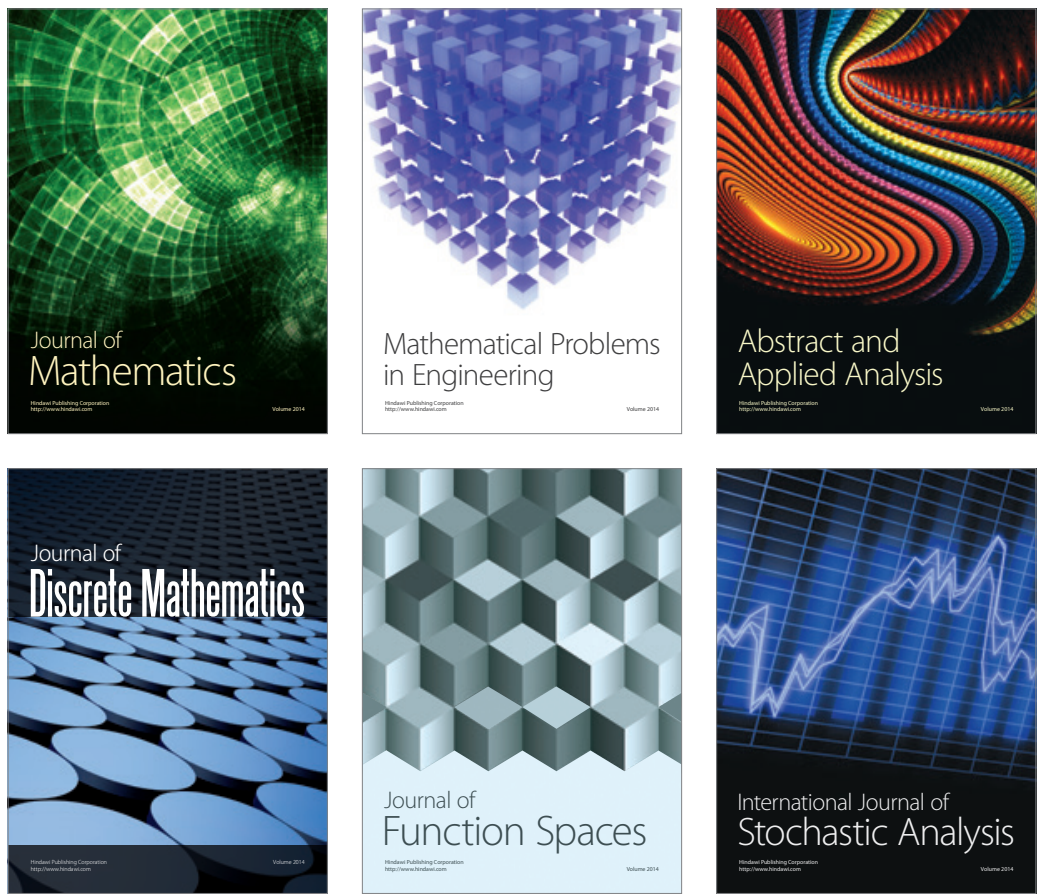

Journal of

Function Spaces

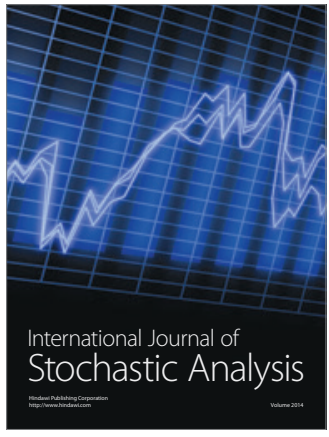

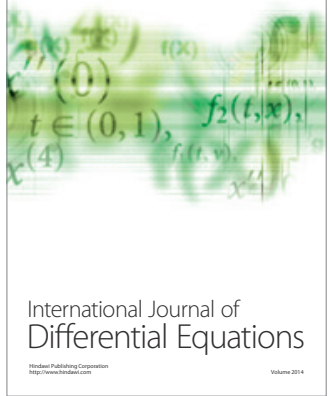
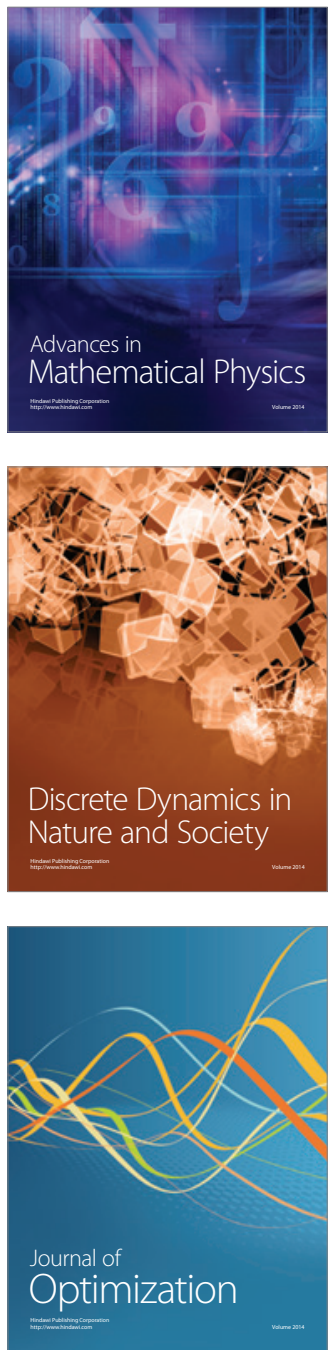